\title{
Summation versus suppression in metacontrast masking: On the potential pitfalls of using metacontrast masking to assess perceptual-motor dissociation
}

\author{
Pedro Cardoso-Leite • Florian Waszak
}

Published online: 11 April 2014

(C) Psychonomic Society, Inc. 2014

\begin{abstract}
A briefly flashed target stimulus can become "invisible" when immediately followed by a mask - a phenomenon known as backward masking, which constitutes a major tool in the cognitive sciences. One form of backward masking is termed metacontrast masking. It is generally assumed that in metacontrast masking, the mask suppresses activity on which the conscious perception of the target relies. This assumption biases conclusions when masking is used as a tool-for example, to study the independence between perceptual detection and motor reaction. This is because other models can account for reduced perceptual performance without requiring suppression mechanisms. In this study, we used signal detection theory to test the suppression model against an alternative view of metacontrast masking, referred to as the summation model. This model claims that target- and maskrelated activations fuse and that the difficulty in detecting the target results from the difficulty to discriminate this fused response from the response produced by the mask alone. Our data support this alternative view. This study is not a thorough investigation of metacontrast masking. Instead, we wanted to point out that when a different model is used to account for the reduced perceptual performance in metacontrast masking, there is no need to postulate a dissociation between perceptual and motor responses to account for the data. Metacontrast masking, as implemented in the Fehrer-Raab situation, therefore is not a valid method to assess perceptual-motor dissociations.
\end{abstract}

\footnotetext{
P. Cardoso-Leite $(\square)$

Faculty of Psychology and Educational Sciences,

University of Geneva, Geneva, Switzerland

e-mail: Pedro.CardosoLeite@unige.ch

F. Waszak

Laboratoire Psychologie de la Perception (UMR 8158), CNRS \&

Université Paris Descartes, Paris, France
}

Keywords Visual awareness · Perception and action · Signal detection theory $\cdot$ Confidence judgments $\cdot$ Reaction times

Visual backward masking has been a major tool in the experimental investigation of visual consciousness during the last decades (Ansorge, Francis, Herzog, \& Oğmen, 2007; for a review, see Breitmeyer \& Ogmen, 2000). It consists in presenting a mask shortly after a target stimulus. The latter is clearly visible when presented in isolation, but its presence may be difficult to report if it is closely followed by the mask.

One particular type of backward masking is called metacontrast masking. In metacontrast masking, the masked stimulus (typically a disk) and the masking stimulus (typically an annulus that covers the disk) share a contour but do not overlap. A standard interpretation of metacontrast masking posits that the mask-induced activations suppress the targetinduced activations (Ansorge, Francis, et al., 2007).

On the basis of this idea, metacontrast masking has been used extensively as an experimental tool to vary the amount of target-related information that can be processed by the brain or to test whether the conscious perception of the target is a necessary prerequisite for other target-related processes (e.g., Lau \& Passingham, 2006; Vorberg, Mattler, Heinecke, Schmidt, \& Schwarzbach, 2003). One example is the Fehrer-Raab effect: Contrary to the target's visibility, simple reaction times (RTs) to a target ("respond as quicky as possible as soon as you detect") are unaffected by metacontrast masking. That is, the target continues to affect RTs, even under experimental conditions in which the mask turns the target (close to) "invisible" (e.g., Bernstein, Amundson, \& Schurman, 1973; Fehrer \& Biederman, 1962; Fehrer \& Raab, 1962; Schiller \& Smith, 1966; Taylor \& McCloskey, 1990; for reviews, see Neumann \& Klotz, 1994; Neumann \& Scharlau, 2007). This result has been interpreted as strong evidence in favor of the independence of perceptual and motor responses 
(e.g., Neumann \& Klotz, 1994; but see also Neumann \& Scharlau, 2007; Waszak, Cardoso-Leite, \& Gorea, 2007; Waszak \& Gorea, 2004; for discussions about which criteria to rely on to make such interpretations, see Cardoso-Leite \& Gorea, 2010; Reingold \& Merikle, 1988; Schmidt \& Vorberg, 2006). Indeed, one intuitive way to explain the Fehrer-Raab effect is to assume that perception occurs after the motor reaction. The neural activations produced by the target may trigger a motor reaction before being affected by the neural activations produced by the mask. The perceptual response, on the other hand, may occur later in time, and thus be affected by mask-related activations (see, e.g., Ogmen, Breitmeyer, \& Melvin, 2003; Waszak et al., 2007). This hypothesis is in line with the idea that the feedforward mask-related signal disrupts the target-related feedback signal from higher to lower visual areas (cf. Di Lollo, Enns, \& Rensink, 2000; Enns \& Di Lollo, 2000; Lamme \& Roelfsema, 2000). It is also in line with the idea that visual signals may be processed along two parallel and interacting channels that differ in their speeds of information transmission and functional roles (Breitmeyer, 1984). In this view, a fast target-related sweep of activity affects the motor response, whereas a slow target-related sweep of activity, which is essential for perception, is suppressed by the fast activity triggered by the mask.

In the present study, we asked participants to react as fast as possible with a simple motor response to a target-mask stimulus. The target was presented on $50 \%$ of the trials; the mask was presented either at the same location as the target (masked) or at a different location (not-masked). At the end of each trial, participants were required to report whether the target was or was not present.

This paradigm allowed us to use signal detection theory (SDT) to test two alternative masking models: a suppression and a summation model (described below in the "Models and predictions" section). Briefly, suppression models posit that the target-related activity is suppressed by the mask. Concerning the Fehrer-Raab effect, this model favors the interpretation of an independence between perceptual and motor responses. The summation model, on the other hand, posits that the target- and mask-related activations are fused. It explains the reduction of perceptual sensitivity by an increased difficulty to discriminate the fused response from the mask-related response, relative to the baseline not-masked condition. Under the summation model, the Fehrer-Raab effect does not imply a dissociation between perceptual and motor responses. These two models make different predictions about the perceptual and motor performances in the masked condition, given the participant's performance in the not-masked condition. Please note that the goal of this study was to test these two classes of metacontrast-masking models, and hence the validity of the conclusion based on the FehrerRaab effect. Our goal was not to present a new model for all forms of backward masking; indeed, the range of parameters tested in this study was very narrow and was specifically selected to study the Fehrer-Raab effect, as observed in a previously tested setting (Waszak et al., 2007). To anticipate, the results of this study support the summation model, and hence put in question the conclusion of a perceptual-motor dissociation based on the Fehrer-Raab effect.

\section{Method}

\section{Observers}

Eight observers, with normal or corrected-to-normal vision and naive as to the purpose of the experiment, participated in this study.

Stimuli

Stimuli were displayed on a $1,024 \times 768$ pixel, $77-\mathrm{Hz}$ color CRT monitor at a viewing distance of $100 \mathrm{~cm}$. The background luminance of the screen was set at $45 \mathrm{~cd} / \mathrm{m}^{2}$. The experiment was programmed and executed using the Psychophysics Toolbox under MATLAB (PTB-2; Brainard, 1997; Pelli, 1997).

During the whole trial, a fixation cross was presented in the center of the screen. The visual stimuli were the target $S_{1}$ and the mask $S_{2}$ (Fig. 1). In present trials (i.e., in half of the trials), $S_{1}$ (which was presented for $13 \mathrm{~ms}$ ) was followed by $S_{2}$ with a stimulus onset asynchrony (SOA) of $52 \mathrm{~ms}$. $S_{2}$ was presented for $39 \mathrm{~ms}$. In absent trials, only $S_{2}$ was presented. $S_{1}$ was a close-to-threshold Gaussian luminance increment with $\sigma=$ $0.269^{\circ}$. The luminance of $S_{1}$ was set to either $10 \%$ or $14 \%$, in the low- or the high-contrast condition, respectively. We decided to use two contrast values instead of only one in order to have an internal replication; the results that are observed when $S_{1}$ has low contrast should not differ qualitatively from the condition in which it has a high contrast. $S_{1}$ was presented $3.7^{\circ}$ below fixation. $S_{2}$ was a highly visible $\left(90 \mathrm{~cd} / \mathrm{m}^{2}\right)$, sharpedged annulus with inner and outer diameters of $0.615^{\circ}$ and $1.153^{\circ}$, respectively. In the masked condition, $S_{2}$ was presented centered on the position of $S_{1}$ (Fig. 1c). In the not-masked condition, $S_{2}$ was presented with the same eccentricity as in the masked condition, but deflected from the vertical meridian by an angle of $22.5^{\circ}$ randomly to the left (not shown) or the right (Fig. 1b).

Tasks and procedures

Figure 1a illustrates the sequence of events in one trial. Observers initiated a new trial with a buttonpress. Each trial started with the onset of a blank screen with only the fixation cross. After a random interval (300-1,000 ms), a stimulus sequence was presented. Observers were required to give 


\section{a)}
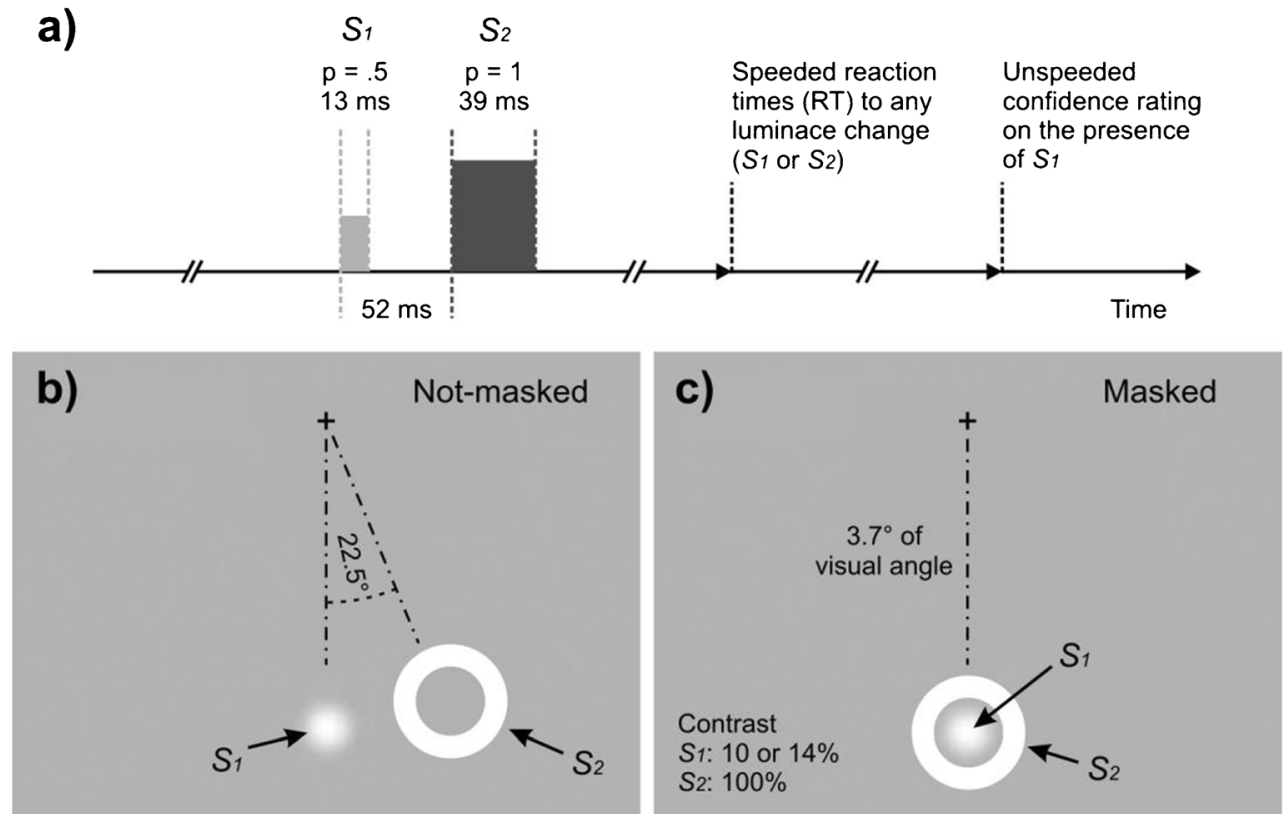

Fig. 1 Stimulus design. (a) Temporal structure of one trial. (b-c) Spatial layouts: $S_{2}$ was centered on $S_{1}$ (masked condition) or displaced randomly to the left or right (not-masked condition)

two responses. First, they pressed a key on the keyboard (the space bar) as quickly as possible when they detected any luminance change (i.e., the onset of $S_{1}$ or $S_{2}$ ). The RT was defined as the latency between this keypress and the possible onset of $S_{1}$ (even if it was not presented). Second, after the first response, observers indicated - without time pressure - their confidence level about the presence or absence of $S_{1}$ by pressing the right ("present") or left ("absent") arrow of the keyboard one to five times (the numbers 1 to 5 appeared in the center of the screen, with 1 meaning very uncertain and 5 absolutely certain). Observers validated their responses by pressing the downward arrow key.

Stimuli were presented in blocks of 300 trials with $S_{1}$ present or absent, randomly interleaved. Four block types resulted from the combination of the two $S_{1}$ contrast values (i.e., $10 \%$ or $14 \%$ ) and the two $S_{2}$ positions (i.e., masked or not-masked condition); each of these block types was presented five times to the participants (for a total of 20 blocks). The order of blocks was randomized across observers. Trials were rejected if RTs were shorter than $100 \mathrm{~ms}$ or beyond two interquartiles from the condition median (masked, notmasked $\times$ low, high contrast, calculated separately for signal-present and signal-absent trials); on average, 1,230 trials were analyzed per condition and per participant.

\section{Data analysis}

Signal detection theory (SDT; Green \& Swets, 1966) describes a situation in which an observer has to detect a stimulus that can either be physically present ("signal trials") or absent ("noise trials"). To perform this task participants have to rely on some internal/neural activity that is a function of the physical stimulus. This internal activity will have a certain probability distribution when the stimulus is physically absent (the internal noise distribution) and it may have a different distribution when the stimulus is physically present (the internal signal distribution). The difference in the shape (i.e., mean and standard deviation, $S D$ ) of the distributions of internal responses elicited in signal trials relative to their distributions in noise trials characterizes detection sensitivity $\left(d_{\mathrm{a}}\right)$, which is defined as the distance between the means of the internal signal and noise distributions in units of "normalized" standard deviation:

$d_{a}=\frac{\mu_{S}-\mu_{N}}{\sqrt{\frac{\sigma_{S}^{2}+\sigma_{N}^{2}}{2}}}=\left(\frac{2}{\sigma_{S}^{2}+\sigma_{N}^{2}}\right)^{1 / 2}\left(\mu_{S}-\mu_{N}\right)$

where $\mu_{\mathrm{S}}, \sigma_{\mathrm{S}}$ and $\mu_{\mathrm{N}}, \sigma_{\mathrm{N}}$ are the mean and $S D$ of, respectively, the internal signal and noise distributions (Macmillan \& Creelman, 2005, p. 62). The higher the $d_{\mathrm{a}}$, the better the perceptual system is able to differentiate signal trials from noise trials.

The decision or response criterion $(c)$ is the value that the internal response has to exceed in order for the participant to report the stimulus to be present. The lower the criterion, the smaller the internal responses need to be for participants to report "stimulus present." In the case of confidence judgments it can be assumed that participants set a series of decision criteria that subdivide the continuum of internal responses in different levels of confidence. 
The mean and $S D$ of the signal and noise distributions (i.e., $\mu_{\mathrm{S}}, \sigma_{\mathrm{S}}$ and $\mu_{\mathrm{N}}, \sigma_{\mathrm{N}}$ in Eq. 1) are unknown parameters. However, since sensitivity is a relative measure, the mean and the $S D$ of the noise distribution can have arbitrary values (by convention, $\mu_{\mathrm{N}}=0, \sigma_{\mathrm{N}}=1$ ); the noise distribution serving as a reference. Given these assumptions, it is straightforward to find the criterion value corresponding for example to the rate of false alarms (i.e., signal is reported present on a noise trial). In this case, $c$ can be defined as the $z$ score (or inverse of the cumulative normal distribution) of 1 minus the false alarm rate $p(\mathrm{FA})$ - that is, $c=\Phi^{-1}[1-p(\mathrm{FA})]$. The same logic is used to locate the criteria underlying the confidence ratings. Once the mean and $S D$ of the noise distribution have been set and the multiple criteria computed on the basis of the response rates on noise trials, it is possible to fit the mean and $S D$ deviation of the signal distribution that best fits the rates of responses within each confidence level on signal trials. By confronting the rates of responses at each confidence level in the signal present and signal absent trials it is possible to infer the (1) difference in mean (i.e., $\mu_{\mathrm{S}}-\mu_{\mathrm{N}}$ ) and (2)the ratio of the $S D$ (i.e., $\sigma_{\mathrm{S}} / \sigma_{\mathrm{N}}$ ) of the distributions of internal responses in signal present trials relative to an arbitrary defined distribution of internal responses in signal absent trials (Green \& Swets, 1966; Macmillan \& Creelman, 2005).

The derivation of the signal distribution from participant's confidence judgments has three advantages. First, in contrast to simple yes/no responses computing sensitivity from confidence judgments yields correct perceptual sensitivity estimates even if signal and noise distributions have different $S D$ s. Second, the model test presented below requires the evaluation of the $S D$ of the signal distribution. Confidence judgments provide an efficient measure for this. Finally, the use of confidence judgments allows the assessment of multiple internal response levels on a continuous scale that can be used to estimate quantitatively the relationship between the internal perceptual response and the observed behavioral response times (see also Cardoso-Leite \& Gorea, 2009).

\section{Models and predictions}

Sensitivity is a relative measure. Conceptually it characterizes a system's capacity to discriminate two situations (e.g., signal and noise trials); formally it is the normalized distance between a signal distribution and a noise distribution of internal responses (see Eq. 1). Although the fact that sensitivity is a relative measure might not be important in some situations (e.g., detecting luminance increments of different amplitudes), not acknowledging it can lead to wrong conclusions in other situations. In the present study we will attempt to show that the detection of metacontrast masked visual stimuli (at least within the limited range of parameters tested in this study) may be part of the latter. We test different predictions of the sensitivity measures in the masked condition relative to the not-masked condition depending on what are assumed to be the noise and signal distributions that underlie the participants' perceptual responses.

Figure 2a shows the probability density functions of the internal responses that are assumed when no signal is present
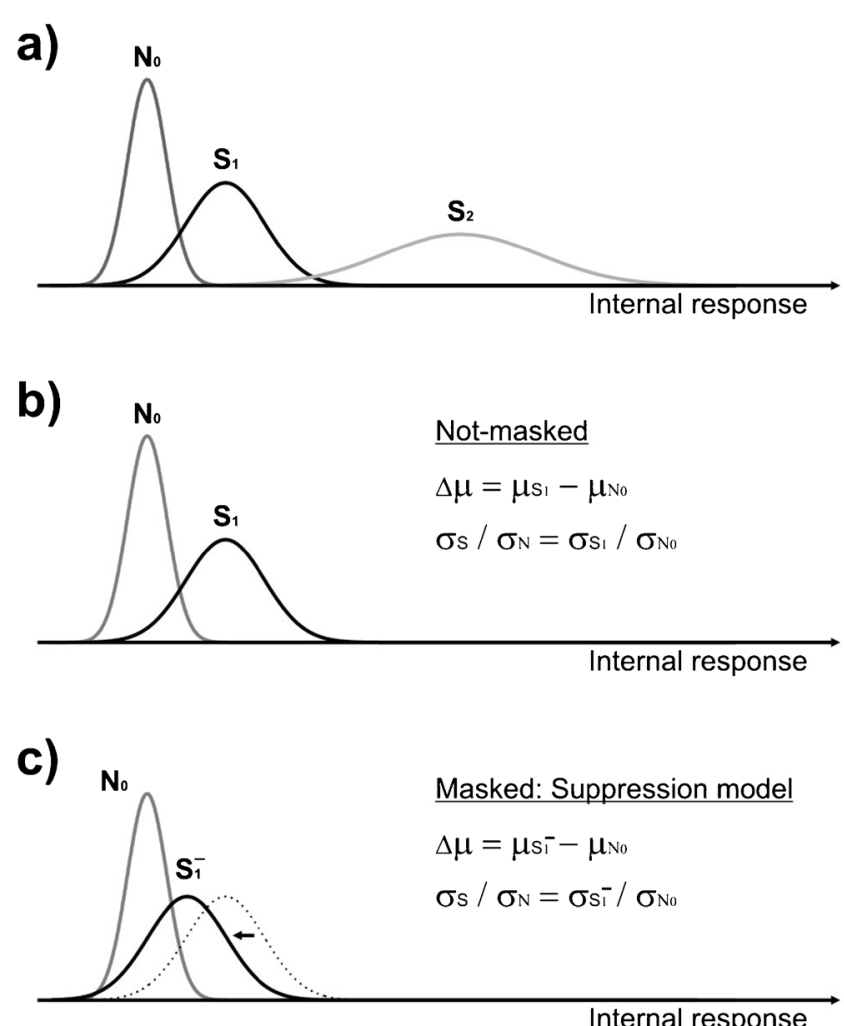

Internal response

c')

Masked: Summation model

$\Delta \mu=\mu s_{1}^{+}-\mu s_{2}$

$\sigma_{s} / \sigma_{N}=\sigma_{s_{1}^{+}}^{+} / \sigma_{s_{2}}$

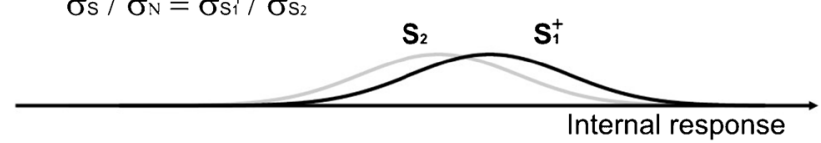

Fig. 2 Signal detection analysis of the effect of metacontrast masking used to make predictions in the present study. (a) Distribution of the "internal responses" (possibly, neural firing rates) in the absence of any stimulation $\left(\mathrm{N}_{0}\right)$, with the presentation of the target only $\left(\mathrm{S}_{1}\right)$, or with the presentation of the mask only $\left(\mathrm{S}_{2}\right)$. The $x$-axis in this figure represents the magnitude of internal responses, implying that increasing a stimulus's intensity should lead to a rightward shift of its internal distribution. (b) Model of the not-masked condition. Participants attempt to discriminate the target-related activity (unaffected by the mask) from the activity observed in the absence of any stimulation $\left(\mathrm{N}_{0}\right)$. Panels $\mathrm{c}$ and $\mathrm{c}^{\prime}$ represent two alternative models of the masked condition: (c) In the suppression model, the activity related to $S_{1}$ is inhibited/suppressed into $S_{1}^{-}$. The $S_{1}{ }_{1}^{-}$ distribution is shifted toward the noise, and possibly flattened, relative to the unaffected $S_{1}$ distribution. (c') Under the summation model, the $\mathrm{N}_{0}$ distribution is irrelevant. The response to $S_{1}$ is added to the response to $S_{2}$ to form $\mathrm{S}_{1}{ }^{+}$. When asked to detect the presence of the $S_{1}$, participants attempt to discriminate $\mathrm{S}_{1}^{+}$from $\mathrm{S}_{2}$ 
$\left(\mathrm{N}_{0}\right)$, when the target $S_{1}$ is presented alone $\left(\mathrm{S}_{1}\right)$, or when the mask $\left(S_{2}\right)$ is presented alone $\left(\mathrm{S}_{2}\right){ }^{1}$ Figure $2 \mathrm{~b}$ illustrates the decision variables that are relevant in the not-masked condition, in which it is assumed that participants detect $S_{1}$ 's presence by discriminating the internal responses caused by $S_{1}$ from those that are observed in the absence of any stimulation - that is, the decision process operates on the internal distributions $\mathrm{S}_{1}$ and $\mathrm{N}_{0} ; \mathrm{S}_{2}$, the mask-related distribution of internal responses, is supposed to be irrelevant for the perceptual task in this condition. Figure $2 \mathrm{c}$ and $\mathrm{c}^{\prime}$ represent two distinct accounts of the decision variables in the masked condition. These two accounts of metacontrast masking (see below) differ regarding the processes that underlie masking, the means and $S D$ s of the signal and noise distributions, and the independence of motor reactions and perceptual judgments. Again, the values of the means and $S D$ s of the distributions depicted in Fig. 2 are not directly accessible; what is accessible, however, are relative measures - that is, (1) the difference in means (i.e., $\mu_{\mathrm{S}}-\mu_{\mathrm{N}}$ ) and (2) the ratio of the $S D$ s (i.e., $\sigma_{\mathrm{S}} / \sigma_{\mathrm{N}}$ ). The predictions of the two masking accounts that are presented below differ in terms of these relative measures (see Fig. 3).

Suppression model (Fig. 2c) Suppression models state that the mask $\left(S_{2}\right)$ reduces the target's visibility $\left(\mathrm{S}_{1}\right)$ by suppressing the activations produced by the target $\left(\mathrm{S}_{1} \rightarrow \mathrm{S}_{1}{ }^{-}\right)$. This suppression could be achieved by decreasing the average targetrelated activity level (a shift of the signal distribution toward the noise distribution) and possibly increasing the spread of the signal distribution. Indeed, if the suppression effect on the target is a noisy process (i.e., the strength of suppression varies across trials), then the resulting $\mathrm{S}_{1}{ }^{-}$would have a larger variance than $\mathrm{S}_{1}$. More formally, the variance ratio under the not-masked condition is given by $\sigma_{\mathrm{S} 1} / \sigma_{\mathrm{N} 0}$, whereas the variance ratio under the suppression model is given by $\left(\sigma_{\mathrm{S} 1}+\right.$ $\left.\sigma_{\text {Suppr }}\right) / \sigma_{\mathrm{N} 0}$, where $\sigma_{\text {Suppr }}$ denotes the variance (larger than or equal to zero) introduced by the suppression mechanism. It is then clear that $\left(\sigma_{\mathrm{S} 1}+\sigma_{\mathrm{Suppr}}\right) / \sigma_{\mathrm{N} 0}$ must be larger than or equal to $\sigma_{\mathrm{S} 1} / \sigma_{\mathrm{N} 0}$. The predictions of the suppression model are illustrated in Fig. 3. Relative to the not-masked condition, the mean of the signal distribution is predicted to decrease (Fig. 3a), and its standard deviation (SD) to increase (Fig. 3b). According to this model, when participants attempt to report the presence of $S_{1}$, they discriminate $\mathrm{S}_{1}^{-}$from $\mathrm{N}_{0}$; as in the not-masked case, the internal response to the mask $\left(\mathrm{S}_{2}\right)$ is not relevant for the perceptual decision.

Summation model (Fig. 2 2') The summation account posits that the internal response evoked by the target $\left(\mathrm{S}_{1}\right)$ is added-

\footnotetext{
${ }^{1}$ Letters in italics refer to the physical stimuli, and plain capitals refer to the corresponding internal random (decision) variables.
}

possibly with some leakage - to the response produced by the mask $\left(\mathrm{S}_{2}\right)$. According to this view, participants' detection task in the masked condition consists in discriminating the response produced jointly by the target and the mask $\left(\mathrm{S}_{1}+\mathrm{S}_{2} \rightarrow \mathrm{S}_{1}{ }^{+}\right)$ from that produced by the mask alone $\left(\mathrm{S}_{2}\right)$. Under this model, the "reference" noise distribution is not $\mathrm{N}_{0}$ (as in the notmasked case, and under the suppression model in the masked condition), but $\mathrm{S}_{2}$. Under the assumption that the summation of $\mathrm{S}_{1}$ and $\mathrm{S}_{2}$ is not perfect (e.g., because of leakage), this model predicts a decrease of the distance between the signal and noise means relative to the not-masked condition (and no difference, if the summation is perfect).

The predictions of the signal $S D$ under the summation model are based on the observation that under masking-free conditions, both the mean and the $S D$ of the distribution of internal responses increase with increasing signal strength (the change in the mean being about four times larger than the change in the $S D$; cf. Nachmias \& Steinmann, 1963; for a review, see Green \& Swets, 1966, chap. 4, p. 95). It follows that the $S D \mathrm{~s}$ of the $\mathrm{S}_{1}{ }^{+}$and $\mathrm{S}_{2}$ distributions will be quite similar, because the contribution to the $S D$ of $\mathrm{S}_{1}^{+}$of the low-intensity $S_{1}$ is much smaller than that of the high-intensity $S_{2}$. As can be seen in Fig. $2 \mathrm{c}^{\prime}, \sigma_{\mathrm{S}}$ and $\sigma_{\mathrm{N}}$ will be much larger in the masked than in the notmasked condition.

More formally, the variance ratio in the masked condition under the summation model is given by $\left(\sigma_{\mathrm{S} 1}+\sigma_{\mathrm{S} 2}\right) / \sigma_{\mathrm{S} 2}-$ because in this model, the target- and mask-related responses are fused and compared to the mask-related activity. Whether this ratio is larger or smaller than the variance ratio under the not-masked condition [i.e., $\sigma_{\mathrm{S} 1} / \sigma_{\mathrm{N} 0}$ ] depends on the values of both $\sigma_{\mathrm{S} 1}$ and $\sigma_{\mathrm{S} 2}$. If $\sigma_{\mathrm{S} 1}$ is larger than 1 and $\sigma_{\mathrm{S} 2}$ grows to infinity, then this ratio will be less than the not-masked ratio. More concretely, if we assume only that $\sigma_{\mathrm{S} 2}$ is larger than $\sigma_{\mathrm{S} 1}$, then the variance ratio in the masked condition under the summation model is guaranteed to be smaller than the variance ratio in the not-masked condition-as long as $\sigma_{\mathrm{S} 1}$ is larger than 2 (as can be seen by plugging in this value for $\sigma_{\mathrm{S} 1}$ and $\sigma_{\mathrm{S} 2}$ in the expression above). If $\sigma_{\mathrm{S} 1}$ is smaller than 2 , it is necessary to make further assumptions about a lower-bound value for $\sigma_{\mathrm{S} 2}$ : If $\sigma_{\mathrm{S} 1}=1, \sigma_{\mathrm{S} 2}$ has to be infinitely large; if $\sigma_{\mathrm{S} 1}=$ $1.25, \sigma_{\mathrm{S} 2}$ has to be larger than 5 ; and if $\sigma_{\mathrm{S} 1}=1.5, \sigma_{\mathrm{S} 2}$ has to be larger than 3 . The average variance ratio observed in the notmasked condition is between 1.75 and 2, implying that $\sigma_{\mathrm{S} 2}$ needs not to be larger than 3 for the summation model to predict a reduced variance ratio relative to the not-masked case. Finally, it should be noted that if the values of $\sigma_{\mathrm{S} 2}$ are smaller than these lower bounds, the summation model would predict an increased variance ratio, and therefore be indistinguishable from the suppression model. If, however, a reduced variance ratio were to be observed, then only the summation model (with adequate parameter values) would be plausible. The predictions of the 


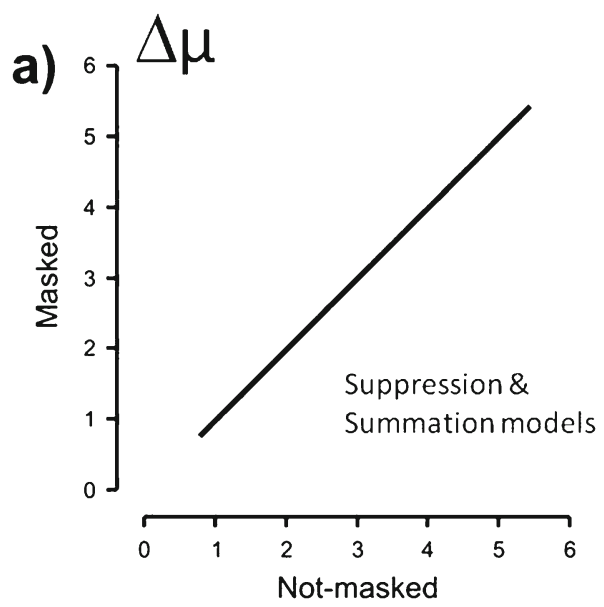

Fig. 3 Predictions made by the summation and suppression models of the relationship between (a) the difference in means between the noise and signal distributions $(\Delta \mu)$ and (b) the signal-over-noise standard deviations ratio $\left(\sigma_{\mathrm{S}} / \sigma_{\mathrm{N}}\right)$ in the masked condition, as a function of the

summation model, assuming that $\sigma_{\mathrm{S} 2}$ is sufficiently large, are illustrated in Fig. 3.

In short, whereas the suppression and summation models may both account for a reduction of the inferred distance between the means of the signal and noise distributions (see Fig. 3a), they do differ on their predictions regarding the change of its standard deviation when comparing notmasked to masked conditions: The suppression account predicts an increase (or no change) of the variance ratio, but a decrease of this ratio could be accounted for only by the summation account (see Fig. 3b).

\section{Results}

This section is subdivided into two parts. First we will analyze the data with respect to the Fehrer-Raab effect, which states that RTs - contrary to the perceptual responses - are not affected by masking. In a second part, we will investigate the perceptual response patterns in order to test the suppression and summation models presented in the Model and Predictions section above.

\section{Fehrer-Raab effect}

RTs and perceptual sensitivity $\left(d_{\mathrm{a}}\right)$ were first analyzed as a function of masking condition (masked, not masked) and $S_{1}$ contrast (10\%, $14 \%$; see Fig. 4). For speeded responses (see Fig. 1a), a two-way repeated measures analysis of variance (rmANOVA) on median RTs on signal-present trials only yielded a significant main effect of contrast $[F(1,7)=$ $\left.32.699, p=7.22 \times 10^{-4}\right]$ - that is, RTs decreased with increasing contrast. Masking had no significant effect on

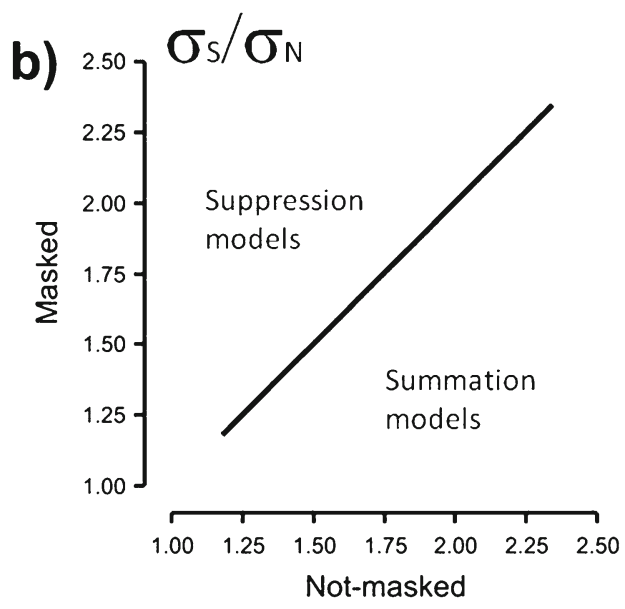

equivalent statistic measured in the not-masked condition. Although both models predict a reduction in $\Delta \mu$, the suppression model predicts an increase in $\sigma_{\mathrm{S}} / \sigma_{\mathrm{N}}$, where the summation model predicts a decrease of this ratio

RTs $[F(1,7)=4.0385, p=.0844]^{2}$ and did not interact with $S_{1}$ contrast $[F(1,7)=0.2987]$. On the other hand, a two-way rmANOVA on $d_{\mathrm{a}}$ yielded significant main effects for both masking condition $[F(1,7)=15.91, p=.005]$ and $\mathrm{S}_{1}$ contrast $[F(1,7)=34.46, p<.001]$, without an interaction $[F(1,7)=1.03, p=.344]$. Hence, these results replicate the phenomenon known as the Fehrer-Raab effect, according to which masking reduces the visibility of a target without altering RTs (Fehrer \& Raab, 1962).

To investigate whether the RT in $S_{1}$-present trials depends on observers correctly reporting the stimulus presence (hit) or not (miss), we computed - for each participant individuallythe difference in median RTs between, on the one hand, perceptual hit and miss trials and, on the other hand, correct rejection (CR) trials (i.e., trials in which $S_{1}$ was both physically absent and correctly reported to be so). A rmANOVA with Masking Condition (masked, not masked), $S_{1}$ Contrast $(10 \%, 14 \%)$, and Perceptual Response (hit, miss) as factors yielded a significant main effect of $S_{1}$ contrast $[F(1,7)=$

\footnotetext{
${ }^{2}$ It is legitimate to question the statistical power of our analysis to show that masking does not affect RTs, especially given the small number of participants in this study. Statistical power depends on both sample size and effect size: For any nonzero effect size, it is always possible to find a sample size that will reveal statistically significant results. In order to estimate an expected effect size of masking on RTs under the hypothesis that masking affects both perception and motor responses, we used the following reasoning: The contrast manipulation yielded a sensitivity change of 0.69 , whereas the masking manipulation yielded a sensitivity change of 0.94 . Contrast changed RTs by $13.1 \mathrm{~ms}$. If masking had equivalent effects on sensitivity and RTs, we would expect a masking effect on RTs of $17.85 \mathrm{~ms}$ [viz. $13.1 *(0.94 / 0.69)=17.85 \mathrm{~ms}$ ]. Note that $17.85 \mathrm{~ms}$ is much larger than the observed effect of contrast on RTs $(13.1 \mathrm{~ms})$. Note also that we had enough statistical power to detect this contrast effect of $13.1 \mathrm{~ms}$. It follows that we should also have had enough power to detect an effect of masking on RTs, had there been such an effect.
} 


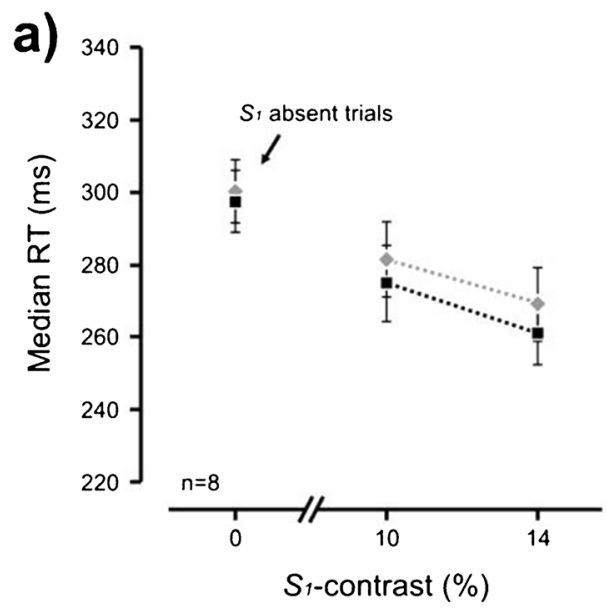

Fig. 4 The Fehrer-Raab effect. Reaction times (RTs, panel a) and sensitivity (b) in the masked and not-masked conditions, as a function of $S_{1}$ contrast. Masking seems to have no significant impact on RTs, but it

36.66, $p=.001]$. Masking condition did not have a significant effect $[F(1,7)=1.38, p=.278]$, nor did it interact with contrast $[F(1,7)<1]$. These findings are in agreement with the previous analysis on median RTs (see Fig. 4). We observed a significant interaction between masking condition and perceptual response $[F(1,7)=7.64, p=.028$; the interaction between perceptual response and contrast, $F(1,7)=1.63, p=$ .243 , as well as the triple interaction, $F(1,7)=2.36, p=.168$, were not significant]. These results indicate that the relationship between RT and perceptual response depends on the masking condition. Most interestingly, we found a significant main effect of perceptual response $[F(1,7)=259.74$, $p<.001]$. Clearly, perceptual and motor responses are not independent.

Finally, on both miss and CR trials, observers indicated that $S_{1}$ was not present, but $S_{1}$ was in fact physically present in the former and absent in the latter case. To test whether RTs were affected by these "unseen" stimuli, we performed one-tailed $t$ tests on the median RT differences (between miss and CR trials), with the null hypothesis being that differences were equal to 0 . RTs were faster on miss than on CR trials, in both the masked condition ( $10 \%$ contrast: $t=-3.634, p=.008$; $14 \%$ contrast: $t=-5.1564, p=.001)$ and the not-masked condition ( $10 \%$ contrast: $t=-2.7575, p=.028 ; 14 \%$ contrast: $t=-4.3849, p=.003$; the Bonferroni-corrected significance level for multiple one-tailed $t$ tests was .025). Hence, in both the masked and unmasked conditions, RTs were affected by stimuli that were reported as unseen. It should be noted that an effect of these unseen stimuli was to be expected, not only under the hypothesis that unconscious stimuli may trigger motor responses, but also if participants by mistake sometimes pressed the key that did not correspond to their perception, if they forgot what their perception was after having made their speeded response, or if they occasionally guessed on a response.

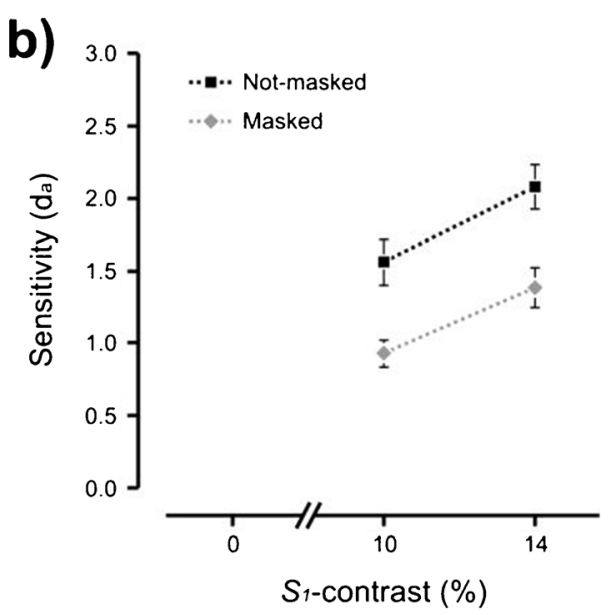

shows a strong effect on sensitivity. A main effect of $S_{1}$ contrast is evident in both the behavioral measures. Data points indicate the means and SEMs of eight observers

\section{Perceptual responses}

Figure 5a presents the mean of the signal distribution inferred from the confidence ratings in the masked condition as a function of the mean of the signal distribution associated with the not-masked condition. Because all data points are below the identity line, it can be concluded that masking reduces the distance between the mean of the signal distribution $\left(\mathrm{S}_{1}{ }^{-}\right.$or $\left.\mathrm{S}_{1}{ }^{+}\right)$and the mean of the reference noise distribution $\left(\mathrm{N}_{0}\right.$ or $\mathrm{S}_{2}$ ). In other words, the difference in means is larger between $\mathrm{S}_{1}$ and $\mathrm{N}_{0}$ (not-masked condition) than between $\mathrm{S}_{1}^{-}$and $\mathrm{N}_{0}$ (masked condition: suppression model) or between $\mathrm{S}_{1}{ }^{+}$and $\mathrm{S}_{2}$ (masked condition: summation model).

This conclusion is confirmed by paired $t$ tests for both the low- and high-contrast stimuli [respectively, $t(7)=5.122$, $p<.002 ; t(7)=7.323, p<.001]$. Note that this result is expected by both accounts of metacontrast masking (see Fig. 3a).

Figure $5 \mathrm{~b}$ shows the standard deviation of the signal distribution inferred from the perceptual responses in the masked condition as a function of the standard deviation of the signal distribution measured in the not-masked condition. Critically, and as we detailed above, the suppression model predicts an increase (or no change) of the standard deviation of the signal distribution. Hence, the data points in this plot should lie above (or along) the main diagonal. The summation model, by contrast, predicts a decrease of the signal's standard deviation, a prediction that would translate into data points being below the identity line. Cleary, the present data support the summation model (see Fig. 3b), since all data points are below the identity line [paired $t$ tests: low contrast, $t(7)=4.88, p<.002$; high contrast, $t(7)=7.587, p<$ .001]. This pattern of results presents strong evidence against the suppression model and in favor of the summation model. 


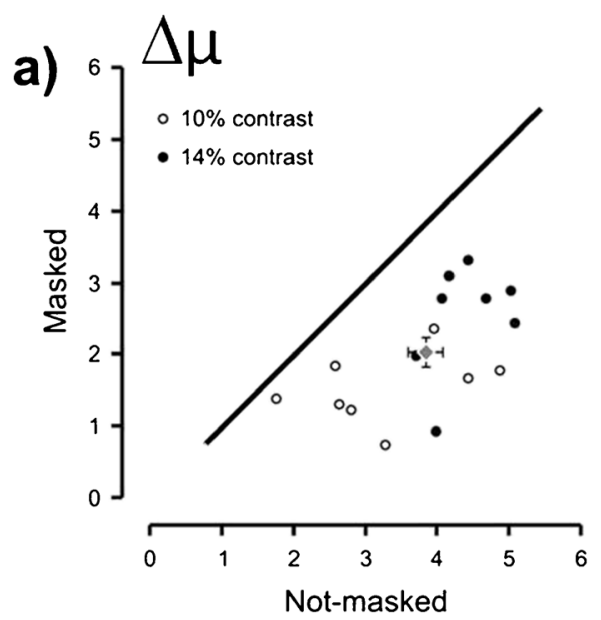

Fig. 5 Means (a) and standard deviations (b) of the signal distribution inferred from the confidence judgments in the masked condition, as a function of the equivalent statistics measured in the not-masked condition. The black and white dots represent the high- and low-contrast

\section{Discussion}

The aim of the present study was to contrast two distinct classes of models of visual metacontrast masking: suppression and summation models. Suppression models comprise accounts assuming that the response to a visual stimulus is suppressed by the subsequently presented mask. This intuitive view corresponds to what is, at least implicitly, assumed when metacontrast masking is used as a tool (e.g., to produce invisible stimuli and test whether perception and action are independent; cf. Ansorge, Francis, et al., 2007). Summation models, by contrast, claim that target- and mask-related brain responses are blended. As a consequence, when asked to detect the target, human observers discriminate this merged activation from the activation triggered by the presentation of the mask in isolation.

These two types of models make different predictions about how the signal distribution will change when the target stimulus is masked relative to when it is not. Both the summation and the suppression models predict that the mean of the signal distribution will be reduced, and thereby explain why metacontrast masking hampers the visibility of the target. Most critically, however, these models differ in terms of their predictions of the variances of the internal distributions on target-present trials. Unfortunately, we do not have direct access to these internal signals, and must rely on the distributions of participants' confidence ratings, which provide only a relative measure. As we outlined above, only under the summation model will metacontrast masking seemingly reduce the variance of the signal distribution. The results of the present study support the summation model.

Under the assumption that the mask suppresses the targetrelated activity, it has been concluded that because perception but not motor reactions are affected by masking (the "Fehrer-

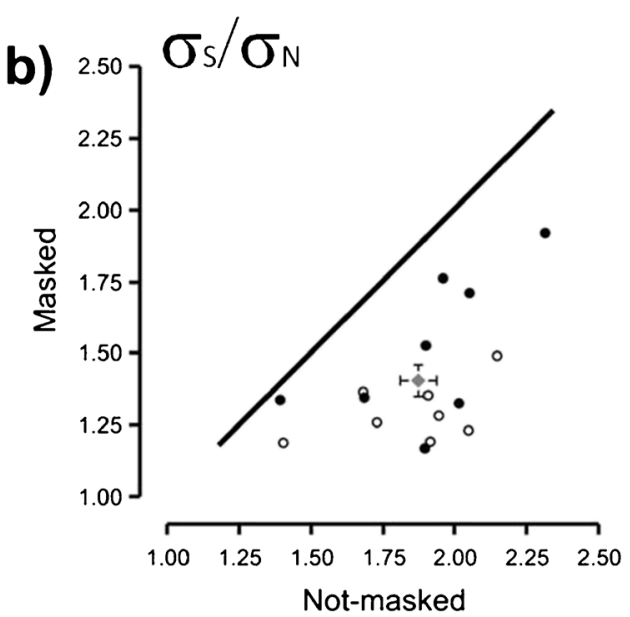

conditions, respectively. Each dot stands for an observer. The cross represents the center of the data points, with the vertical and horizontal error bars indicating SEMs

Raab" effect), perception and action are independent (for a review, see Neumann \& Klotz, 1994; but see also Neumann \& Scharlau, 2007; Waszak et al., 2007; Waszak \& Gorea, 2004). By rejecting the suppression model in favor of the alternative summation model, the present data also cast doubts as to whether or not the perceptual and motor responses dissociate in this type of experiment.

Numerous models have been devised to account for perceptual performance across a variety of backward-masking situations (for a review, see Breitmeyer \& Ogmen, 2000), the most popular ones being perhaps the dual-channel model (Breitmeyer, 1984) and the recurrent-processing model (Enns \& Di Lollo, 2000). The dual-channel (or transientsustained) model posits that visual information is processed along two distinct but interacting channels: a fast/transient and a slow/sustained channel. The fast channel is involved in localization and motion perception, whereas the slow channel is involved in color and brightness perception. According to this view, visual backward masking is the result of the slow response to the target being inhibited by the fast response to the subsequently presented mask.

The recurrent-processing model, on the other hand, posits that perception involves a series of iterative processing loops. Visual information is first fed forward from lower to higher visual areas, and then fed back to early visual cortex in order to "confirm" the visual input. In this view, the target's visibility is hampered because the presentation of the mask disrupts/ interferes with the processing of the target when the feedforward response to the mask meets the feedback signal of the target in early visual areas. Before the feedforwardfeedback loop is closed, the target has already been replaced by the mask. These are just two views on metacontrast masking, among numerous others (e.g., the perceptual retouch theory [Bachmann, 1984] and its updated version [Kirt \& 
Bachmann, 2013], to name only a few). The interested reader should consult Breitmeyer and Ogmen (2000) for a review. Since these models focus mainly on how perception varies depending on different stimulus parameters (e.g., SOA or stimulus polarity), it is not clear to us how to derive predictions for RTs from them. Similarly, in the response-priming literature (which uses an approach conceptually different from ours), models have been proposed to account for masked primes affecting motor responses (e.g., Schmidt, Niehaus, \& Nagel, 2006; Vorberg et al., 2003). These models focus on explaining the "unconscious" motor responses, and not so much on explaining how masking affects perception (e.g., Vorberg et al., 2003), or they refer to existing metacontrast models such as the recurrent-processing model described above (e.g., Schmidt et al., 2006).

Although it is tempting to consider the recurrentprocessing model and the dual-channel model to be of the suppression type, we refrain from doing so. The present experimental setting produced a qualitatively new set of data that have not yet been confronted to simulations of the existing models, which might be amended so as to account for them. Future studies will show whether these models can or cannot account for this pattern of results. The naïve summation view presented herein is in perfect agreement with the present data, but it does not by itself account for the variety of experimental results involving metacontrast masking that can be accurately described by suppression-like models (e.g., masking functions with varying SOAs and target and mask intensities). Indeed, the present model might completely fail if we were to choose different experimental settings. However, what this study shows is that, at least within the specifics of the present experimental design, it is possible to model metacontrast masking such that the dissociation observed between perceptual and motor responses does not imply a dissociation of the signals that feed those responses.

This study is not the first to show that target and mask interact in more complex ways than is generally assumed. In line with the summation model, masked targets have been shown to affect the perception of the mask (Ansorge, Becker, \& Breitmeyer, 2009; Ansorge, Breitmeyer, \& Becker, 2007; Herzog \& Koch, 2001; Otto, Oğmen, \& Herzog, 2006; Scharlau \& Neumann, 2003; for reviews, see Ansorge et al., 2009; Herzog, 2007; Scharlau, 2007; Scharlau, Ansorge, \& Breitmeyer, 2006): If, for example, two parallel not-aligned bars are masked by a set of aligned bars, the masking bars are perceived as misaligned: Properties of the masked stimulus can thus be inherited by the mask. More generally, by using different target and mask configurations, these studies have emphasized the importance of taking into account the spatial and temporal interactions between stimuli presented in close succession. The present study extends our knowledge by showing that the visibility of the target is hampered at least partly because of a change of the internal reference against which target-related activations are compared.

Currently, researchers are showing increasingly interest in the notion of "criterion content" introduced by Kahneman (1968). Criterion content refers to the idea that stimuli result in multidimensional percepts. According to his notion, in order to make decisions about these stimuli, participants can rely on different attributes. For example, Lau and Passingham (2006) introduced the concept of relative blindsight after observing that, for two SOAs yielding the same detection accuracy, participants reported different levels of confidence in their responses. However, Jannati and Di Lollo (2012) demonstrated that this perceptualperceptual discrepancy (between detection and confidence) doesn't reflect a fundamental dissociation (for further examples, see Cardoso-Leite, Mamassian \& Gorea, 2009; Cardoso-Leite et al., 2007). Instead, participants rely on different content criteria, depending on the SOA. This result is in line with a pioneering study that used multidimensional scaling to reveal the perceptual dimensions that underlie participants' decisions in a metacontrast detection task (Sackur, 2013). This study showed that at least three dimensions determine participants' judgments: one of time, and two of visibility. Most likely, at short SOAs participants consider the target-mask pair as a unique stimulus, whereas at longer SOAs the target and mask are perceived as two distinct events.

We believe that the largely accepted dissociation between perceptual and motor responses in metacontrast may equally well be explained by criterion content differences across these two response modalities. It seems plausible that participants in our experiment built a unitary but complex percept of the target-mask pair - see also Sackur's (2013) results showing that instructions have little effect on participants' decisions about the target's presence - and that speeded RTs and perceptual detection of the same complex perceptual event rely on different content criteria. It would be interesting to test this hypothesis directly by using multidimensional-scaling methods on both types of behavioral outcomes.

The idea that target-related brain activations must be suppressed under metacontrast masking is appealing because it seems intuitive: After all, a highly visible stimulus is turned invisible by a mask! A similarly mysterious (though different) situation was encountered in the early days of psychophysics: A 10-g heavy weight placed on the palm of the hand can be perfectly detected by itself, but if the task is to discriminate $1,000 \mathrm{~g}$ from $1,010 \mathrm{~g}$, the task is far more difficult, although the absolute difference in weights is the same; yet, one would not assume that the 1,000-g part of the 1,010-g weight masks the remaining $10 \mathrm{~g}$ ! Suppression is not a logical necessity to account for reduced perception, and in the context of the study of the relationship between perceptual and motor responses, assuming the contrary may lead to wrong conclusions.

In the present study, we made a series of assumptions to devise two categories of metacontrast-masking models and to 
test which of these two models would be supported by experimental data. Qualitatively, all predictions were met for the summation model, which could account for an apparent dissociation between perceptual and motor responses, despite there being no genuine differences between the signals on which they relied. Quantitatively, however, we lack a computational model to determine with more certainty if these behavioral results imply that perceptual and motor processes do not dissociate, or if instead other accounts remain possible. Our models rely on multiple assumptions, and the reader may of course question their validity and thus disregard the fulfilled predictions - for example, because we used only a very limited and specific set of parameters, whereas a vast literature has gone against this view. But even in that case, one should keep in mind that conclusions about the dissociation between perceptual and motor responses in metacontrast-masking studies also depends on assumptions of what metacontrast masking does to the visual system, and those assumptions might not always be true.

Author note We thank Andrei Gorea for discussions that led to the idea of this experiment, and Thomas Otto for helpful comments on the manuscript. P.C.-L. was supported by a Fyssen Foundation postdoctoral fellowship. The research leading to these results has received funding from the European Research Council (ERC) under the European Union's Seventh Framework Programme (FP7/2007-2013)/ERC Grant Agreement No. 263067.

\section{References}

Ansorge, U., Becker, S. I., \& Breitmeyer, B. (2009). Revisiting the metacontrast dissociation: Comparing sensitivity across different measures and tasks. Quarterly Journal of Experimental Psychology, 62, 286-309. doi:10.1080/17470210801908492

Ansorge, U., Breitmeyer, B. G., \& Becker, S. I. (2007). Comparing sensitivity across different processing measures under metacontrast masking conditions. Vision Research, 47, 3335-3349. doi:10.1016/j. visres.2007.09.009

Ansorge, U., Francis, G., Herzog, M. H., \& Oğmen, H. (2007). Visual masking and the dynamics of human perception, cognition, and consciousness: A century of progress, a contemporary synthesis, and future directions. Advances in Cognitive Psychology, 3, 1-8. doi:10.2478/v10053-008-0009-0

Bachmann, T. (1984). The process of perceptual retouch: Nonspecific afferent activation dynamics in explaining visual masking. Perception \& Psychophysics, 35, 69-84.

Bernstein, I. H., Amundson, V. E., \& Schurman, D. L. (1973). Metacontrast inferred from reaction time and verbal report: Replication and comments on the Fehrer-Biederman experiment. Journal of Experimental Psychology, 100, 195-201.

Brainard, D. H. (1997). The psychophysics toolbox. Spatial Vision, 10, 433-436. doi:10.1163/156856897X00357

Breitmeyer, B. G. (1984). Visual masking: An integrative approach. New York, NY: Oxford University Press.

Breitmeyer, B. G., \& Ogmen, H. (2000). Recent models and findings in visual backward masking: A comparison, review, and update.
Perception \& Psychophysics, 62, 1572-1595. doi:10.3758/ BF03212157

Cardoso-Leite, P., \& Gorea, A. (2009). Comparison of perceptual and motor decisions via confidence judgments and saccade curvature. Journal of Neurophysiology, 101, 2822-2836. doi:10.1152/jn. 91269.2008

Cardoso-Leite, P., \& Gorea, A. (2010). On the perceptual/motor dissociation: A review of concepts, theory, experimental paradigms and data interpretations. Seeing and Perceiving, 23, 89-151.

Cardoso-Leite, P., Gorea, A., \& Mamassian, P. (2007). Temporal order judgment and simple reaction times: Evidence for a common processing system. Journal of Vision, 7(6), 11. doi:10.1167/7.6.11

Cardoso-Leite, P., Mamassian, P., \& Gorea, A. (2009). Comparison of perceptual and motor latencies via anticipatory and reactive response times. Attention, Perception, \& Psychophysics, 71, 82-94. doi:10.3758/APP.71.1.82

Di Lollo, V., Enns, J. T., \& Rensink, R. A. (2000). Competition for consciousness among visual events: The psychophysics of reentrant visual processes. Journal of Experimental Psychology: General, 129, 481-507. doi:10.1037/0096-3445.129.4.481

Enns, J. T., \& Di Lollo, V. (2000). What's new in visual masking? Trends in Cognitive Sciences, 4, 345-352. doi:10.1016/S1364-6613(00) 01520-5

Fehrer, E., \& Biederman, I. (1962). A comparison of reaction time and verbal report in the detection of masked stimuli. Journal of Experimental Psychology, 64, 126-130.

Fehrer, E., \& Raab, D. (1962). Reaction time to stimuli masked by metacontrast. Journal of Experimental Psychology, 63, 143-147.

Green, D. M., \& Swets, J. A. (1966). Signal detection theory and psychophysics. New York, NY: Wiley.

Herzog, M. H. (2007). Spatial processing and visual backward masking. Advances in Cognitive Psychology, 3, 85-92. doi:10.2478/v10053008-0016-1

Herzog, M. H., \& Koch, C. (2001). Seeing properties of an invisible object: Feature inheritance and shine-through. Proceedings of the National Academy of Sciences, 98, 4271-4275.

Jannati, A., \& Di Lollo, V. (2012). Relative blindsight arises from a criterion confound in metacontrast masking: implications for theories of consciousness. Consciousness and Cognition, 21, 307-314.

Kahneman, D. (1968). Method, findings, and theory in studies of visual masking. Psychological Bulletin, 70, 404-425. doi:10.1037/ h0026731

Kirt, T., \& Bachmann, T. (2013). Perceptual retouch theory derived modeling of interactions in the processing of successive visual objects for consciousness: Two-stage synchronization of neuronal oscillators. Consciousness and Cognition, 22, 330-347.

Lamme, V. A. F., \& Roelfsema, P. R. (2000). The distinct modes of vision offered by feedforward and recurrent processing. Trends in Neurosciences, 23, 571-579. doi:10.1016/S0166-2236(00)01657-X

Lau, H. C., \& Passingham, R. E. (2006). Relative blindsight in normal observers and the neural correlate of visual consciousness. Proceedings of the National Academy of Sciences, 103, 1876318768. doi:10.1073/pnas.0607716103

Macmillan, N. A., \& Creelman, C. D. (2005). Detection theory: A user's guide (2nd ed.). Mahwah, NJ: Erlbaum.

Nachmias, J., \& Steinman, R. M. (1963). Study of absolute visual detection by the rating-scale method. Journal of the Optical Society of America, 53, 1206-1213.

Neumann, O., \& Klotz, W. (1994). Motor responses to nonreportable, masked stimuli: Where is the limit of direct parameter specification? In C. Umiltà \& M. Moscovitch (Eds.), Attention and performance $X V$ : Conscious and nonconscious information processing (pp. 123150). Cambridge, MA: MIT Press.

Neumann, O., \& Scharlau, I. (2007). Experiments on the Fehrer-Raab effect and the "Weather Station Model" of visual backward masking. Psychological Research, 71, 667-677. 
Ogmen, H., Breitmeyer, B. G., \& Melvin, R. (2003). The what and where in visual masking. Vision Research, 43, 1337-1350. doi:10.1016/ S0042-6989(03)00138-X

Otto, T. U., Oğmen, H., \& Herzog, M. H. (2006). The flight path of the phoenix - The visible trace of invisible elements in human vision. Journal of Vision, 6(10), 1079-1086. doi:10.1167/6.10.7

Pelli, D. G. (1997). The VideoToolbox software for visual psychophysics: Transforming numbers into movies. Spatial Vision, 10, 437-442. doi: $10.1163 / 156856897 X 00366$

Reingold, E. M., \& Merikle, P. M. (1988). Using direct and indirect measures to study perception without awareness. Perception \& Psychophysics, 44, 563-575. doi:10.3758/BF03207490

Sackur, J. (2013). Two dimensions of visibility revealed by multidimensional scaling of metacontrast. Cognition, 126, 173-180.

Scharlau, I. (2007). Temporal processes in prime-mask interaction: Assessing perceptual consequences of masked information. Advances in Cognitive Psychology, 3, 241-255. doi:10.2478/v10053-008-0028-x

Scharlau, I., Ansorge, U., \& Breitmeyer, B. G. (2006). Trends and styles in visual masking. Advances in Cognitive Psychology, 2, 1-5.

Scharlau, I., \& Neumann, O. (2003). Perceptual latency priming by masked and unmasked stimuli: Evidence for an attentional interpretation. Psychological Research, 67, 184-196. doi:10.1007/s00426002-0116-3
Schiller, P. H., \& Smith, M. C. (1966). Detection in metacontrast. Journal of Experimental Psychology, 71, 32-39.

Schmidt, T., Niehaus, S., \& Nagel, A. (2006). Primes and targets in rapid chases: Tracing sequential waves of motor activation. Behavioral Neuroscience, 120, 1005-1016. doi:10.1037/07357044.120.5.1005

Schmidt, T., \& Vorberg, D. (2006). Criteria for unconscious cognition: Three types of dissociation. Perception \& Psychophysics, 68, 489504. doi:10.3758/BF03193692

Taylor, J. L., \& McCloskey, D. I. (1990). Triggering of preprogrammed movements as reactions to masked stimuli. Journal of Neurophysiology, 63, 439-446.

Vorberg, D., Mattler, U., Heinecke, A., Schmidt, T., \& Schwarzbach, J. (2003). Different time courses for visual perception and action priming. Proceedings of the National Academy of Sciences, 100, 6275-6280.

Waszak, F., Cardoso-Leite, P., \& Gorea, A. (2007). Perceptual criterion and motor threshold: A signal detection analysis of the relationship between perception and action. Experimental Brain Research, 182, 179-188. doi:10.1007/s00221-007-0984-2

Waszak, F., \& Gorea, A. (2004). A new look on the relation between perceptual and motor responses. Visual Cognition, 11, 947-963. 